\title{
CARACTERÍSTICAS FÍSICO-QUÍMICAS E SENSORIAIS DE GELEIAS DE JAMBOLÃO
}

\author{
MILENE TEIXEIRA BARCIA* \\ ALINE LISBÔA MEDINA* \\ RUI CARLOS ZAMBIAZI**
}

\begin{abstract}
O objetivo deste trabalho foi aproveitar o fruto de jambolão para a elaboração de geleias tradicional e light e avaliar a estabilidade físico-química e sensorial dos produtos durante o período de estocagem de 60 dias. Nas formulações de geleias light foram utilizados quatro tipos de edulcorantes, isolados e combinados: sacarina, ciclamato, acessulfame e esteviosídeo. Logo após o processamento foram realizadas as análises de fluidez, umidade, gordura, proteínas, fibras, cinzas, açúcares totais e redutores, teor de sólidos solúveis, $\mathrm{pH}$ e acidez. Ao final do período de armazenamento foram realizadas as análises de umidade, açúcares totais e redutores, teor de sólidos solúveis, $\mathrm{pH}$ e acidez. As características físico-químicas de todas as geleias enquadraram-se nos padrões da legislação brasileira e se mantiveram estáveis durante todo o período de armazenamento. Sensorialmente, as formulações light não se apresentaram estáveis apenas quanto aos atributos de doçura e consistência. A utilização de diferentes edulcorantes e de suas combinações não afetou a qualidade sensorial das geleias light.
\end{abstract}

PALAVRAS-CHAVE: JAMBOLÃO; GELEIA LIGHT; GELEIA DE JAMBOLÃO.

* Mestrandas em Ciência e Tecnologia Agroindustrial, Faculdade de Agronomia Eliseu Maciel, Universidade Federal de Pelotas (UFPel), Pelotas, RS (e-mails:milenebarcia@ig.com.br;alinemedina@ig.com.br).

** Professor, PhD. Food and Nutritional Science, Departamento de Ciência dos Alimentos, UFPel, Pelotas, RS (e-mail:zambiazi@gmail.com.br). 


\section{INTRODUÇÃO}

O jambolão (Syzygium cumini), árvore de grande porte, pertence à família das Mirtáceas, que frutifica no período de janeiro a maio (MORTON, 1987). Os frutos do jambolão, apesar de um pouco adstringentes, são considerados agradáveis ao paladar mas não se destacam em relação ao sabor especial de tantas outras Mirtáceas brasileiras. Pequenos, de coloração roxa quase negra por fora e de polpa pouco caldosa, o fruto do jambolão envolve caroço único e grande quando comparado ao seu tamanho total (ÁRVORES..., 2005).

Os frutos do jambolão apresentam inconveniente durante seu manuseio pois, devido à forte pigmentação de sua polpa e casca, deixam manchas indeléveis na pele das mãos, nos tecidos das roupas, nos calçamentos das ruas e na pintura dos automóveis (CORRÊA, 1984).

A utilização dos frutos de jambolão para a elaboração de subprodutos tem sido pouco explorada. A região sul do estado do Rio Grande do Sul caracteriza-se como pólo de alimentos, devido a sua linha de produtos à base de frutas e vegetais, como geleias, doces em massa, compotas e sucos. Vários incentivos vêm sendo ofertados para promover a utilização de pequenos frutos nessa região, inclusive de matérias-primas não convencionais. Nesse contexto, o fruto de jambolão poderia ser aproveitado para a elaboração de doces regionais característicos, como geleias convencionais e light.

A demanda por alimentos light estimulou o uso de frutas como ingredientes, pois permite a obtenção de produtos com baixo valor calórico e características semelhantes aos convencionais (CAMPOS e CÂNDIDO, 1995). O mercado de produtos light tem aumentado de forma marcante, impulsionado por grande número de consumidores preocupados com a saúde (SALGADO, 2001).

A geleia, produto preparado com frutas e/ou sucos ou extratos aquosos das mesmas, pode apresentar frutas inteiras, partes e/ou pedaços sob variadas formas, devendo os ingredientes ser misturados com açúcares, com ou sem adição de água, pectina, ácidos e outros compostos permitidos pela legislação. A mistura deve ser convenientemente processada até atingir consistência semi-sólida adequada e, finalmente, acondicionada de forma a assegurar sua perfeita conservação (BRASIL,1978). Na elaboração de geléias light são utilizados edulcorantes para repor a doçura característica do produto. A pectina empregada na elaboração de geleias deve apresentar baixo teor de metoxilação (BTM) para formar gel na presença de íons metálicos bivalentes (normalmente o cálcio), não sendo necessária a presença de grande quantidade de açúcares. A pectina de alta metoxilação (ATM), utilizada na formulação de geleias convencionais, necessita de teores de sólidos solúveis superiores a $50 \%$, além de meios com baixo valor de $\mathrm{pH}$.

Vários são os edulcorantes permitidos para o uso em alimentos e bebidas com baixo valor calórico, todos apresentando características específicas de intensidade, persistência do gosto doce e presença ou não de gosto residual. Para que os edulcorantes sejam aplicados com êxito é necessário que, além de sua segurança absoluta, apresentem características sensoriais agradáveis, doçura semelhante à da sacarose sem conferir sabor residual estranho. Dentre os edulcorantes permitidos pela legislação brasileira destacam-se: a estévia, ciclamatos, sacarina e acessulfame (BRASIL, 2008). O extrato de folhas de estévia (Stevia rebaudiana, Bert.), composto por glicosídeos terpênicos, consiste em pó branco (formado por cristais de estévia), denominado de esteviosídeo, o qual é isento de calorias (WELLS, 1989). A sacarina sódica constitui um dos edulcorantes mais utilizados em alimentos, parte em função de seu preço que é cerca de 20 vezes inferior ao da sacarose e pelo baixo residual apresentado. Não é metabolizado pelo organismo humano, portanto não fornece calorias (HIGGINBOTHAM, 1983). O ciclamato de sódio ou potássio apresenta a vantagem de reduzir o gosto amargo residual da sacarina, quando a ela associado, sendo também isento de calorias por não ser metabolizado pelo organismo humano (WELLS, 1989). O acessulfame-k apresenta diversas características desejáveis, como boa estabilidade em elevadas temperaturas e em baixos valores de $\mathrm{pH}$. Além disso, é considerado seguro para o consumo humano (PARKE et al.,1999). 
O objetivo deste estudo foi de utilizar o fruto de jambolão para a elaboração de geleias tradicional e light e avaliar a estabilidade físico-química e sensorial dos produtos durante 60 dias de estocagem .

\section{MATERIAL E MÉTODOS}

\subsection{MATÉRIA-PRIMA}

Os frutos do Jambolão (Syzygium cumini) foram colhidos no campus da Universidade Federal de Pelotas (UFPel), no Rio Grande do Sul, safra 2006/2007. Após a retirada dos cálices realizouse a seleção e classificação dos frutos segundo a presença de injúrias mecânicas, sanidade e maturação por análise visual. Os frutos foram lavados em água corrente por 2 minutos e deixados para escorrer o excesso de água sobre tecidos de algodão. Parte dos frutos foi utilizada para a elaboração das geleias, sendo a outra porção acondicionada em embalagens de polietileno sob congelamento $\left(-18^{\circ} \mathrm{C}\right)$ até o momento das análises. Foram usadas nas formulações a sacarose comercial, edulcorantes (marca Chemax), pectina de alta metoxilação (ATM com 150 SAG), pectina de baixa metoxilação amidada (BTM), ambas marca CPKelco, ácido cítrico p.a., cloreto de cálcio p.a. e benzoato de sódio p.a.

\subsection{PROCESSAMENTO DAS GELEIAS DE JAMBOLÃO}

Efetuou-se a extração do suco pela cocção do fruto em água fervente, utilizando 2 partes de fruto para 1 parte de água $(\mathrm{p} / \mathrm{v})$, sob aquecimento até atingir a concentração de $10^{\circ}$ Brix. Após o resfriamento, efetuou-se a filtração para a remoção do suco. Depois da definição das formulações com base em testes preliminares(Tabela 1), os ingredientes foram misturados ao suco, procedendose a cocção em tacho aberto de aço inoxidável com agitação manual contínua até a concentração final de sólidos solúveis de $73^{\circ}$ Brix para a geleia tradicional e de $48^{\circ}$ Brix para as geleias light. Seguiu-se $o$ envase a quente em embalagens de vidro, previamente esterilizadas (capacidade de $250 \mathrm{~g}$ ), vedadas manualmente com tampa de metal. Os frascos foram invertidos por 30 min e armazenados à temperatura ambiente em local isento da incidência direta de luz.

\subsection{AVALIAÇÕES FÍSICO-QUÍMICAS}

Logo após o processamento (tempo zero) das geleias e aos 30 e 60 dias de armazenamento, foram realizadas as análises físico-químicas de fluidez, umidade, carboidratos por diferença, açúcares totais, açúcares redutores, açúcares não redutores, teor de sólidos solúveis ( ${ }^{\circ}$ brix), pH e acidez. Após o processamento também foram realizadas as análises de gordura, proteínas, fibras e cinzas.Todas as análises foram efetuadas em triplicata, conforme as metodologias do Instituto Adolfo Lutz (1985).

O teor de carboidratos e o valor calórico das geleias foram determinados segundo a RDC $\mathrm{n}^{\circ}$ 360, de dezembro de 2003 (BRASIL, 2003).

\subsection{AVALIAÇÃO SENSORIAL}

A avaliação sensorial dos produtos foi efetuada logo após o processamento e aos 30 e 60 dias de armazenamento. Todas as avaliações foram realizadas em cabines individuais, providas de luz fluorescente, do Laboratório de Análise Sensorial de Alimentos do Departamento de Ciência dos Alimentos da Universidade Federal de Pelotas UFPel). 


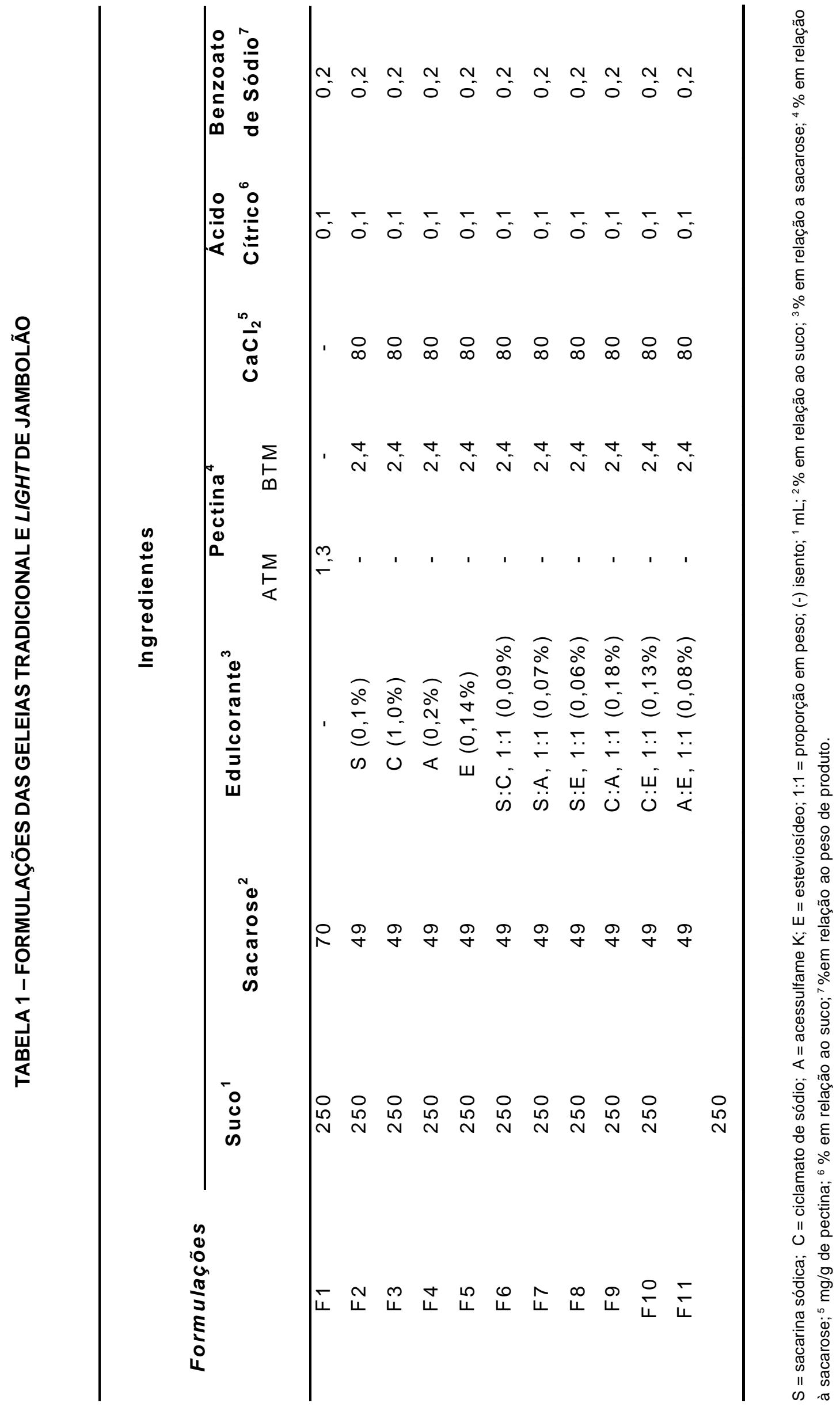


Quinze julgadores, previamente selecionados, foram treinados mediante testes de gostos doce, ácido, metálico e amargo, e de consistência do gel. Para o teste de gostos foram preparadas soluções com diferentes concentrações de sacarose (doce), ácido cítrico (ácido), sulfato de ferro (metálico) e cafeína (amargo), segundo procedimentos descritos pela ABNT (1993). Para a avaliação de consistência foram preparadas geleias com diferentes consistências (de mole à dura). $O$ treinamento da equipe continuou até o momento em que os julgadores atingiram pelo menos $70 \%$ de acertos nos testes.

Combinações aleatórias das amostras foram apresentadas aos julgadores, em recipientes descartáveis brancos, codificados com algarismos de três dígitos, contendo cerca de $20 \mathrm{~g}$ de geleia à temperatura ambiente. Para as avaliações das geleias foram empregadas escalas não estruturadas de $9 \mathrm{~cm}$, segundo indicação de GULARTE (2002).

O julgador devia assinalar na ficha o ponto que descrevesse a intensidade percebida para os atributos de cor roxa (1 - roxo claro a 9 - roxo escuro), consistência (1 - líquida a 9 - muito consistente), acidez (1 - imperceptível a 9 - muito intenso), doçura (1 - imperceptível a 9 - muito intenso) e sabor residual (1 - imperceptível a 9 - muito intenso).

A avaliação da aparência das amostras (cor e brilho) foi conduzida sob luz branca e a dos demais atributos sob luz vermelha para evitar que diferenças na aparência das amostras pudessem influir na resposta dos julgadores (GULARTE, 2002).

\subsection{ANÁLISE DOS DADOS}

Os dados obtidos foram avaliados pela análise de variância e para os resultados significativos, as médias foram comparadas pelo teste de Tukey (ao nível de $5 \%$ de probabilidade), usando-se o programa Statistica 7.0 (STATSOFT, 2008).

\section{RESULTADOS E DISCUSSÃO}

\subsection{DETERMINAÇÕES FÍSICO-QUÍMICAS NAS GELEIAS}

Mesmo apresentando pequenas variações, os teores de proteínas, gorduras, fibras e cinzas (Tabela 2) são característicos de geleias regionais de frutas, assemelhando-se aos das geleias comerciais.

O teor de umidade da geleia tradicional (27\%) mostrou-se significativamente superior ao das geleias light (média de 46\%), que se justifica pela maior concentração ('Brix) final da geleia tradicional. Observou-se pequena variabilidade no teor de umidade entre as geleias light em função do ${ }^{\circ}$ Brix final dessas formulações.

De acordo com os dados das análises físico-químicas todas as geleias light apresentaram redução calórica superior a 25\% (25,9 a 37,9\%) em relação à geleia tradicional, atendendo ao percentual mínimo de redução estipulado pela legislação (BRASIL, 1978) para o produto ser considerado light (Figura 1).

Os valores calóricos das geleias light de jambolão variaram entre 175 a $209 \mathrm{kcal} / 100 \mathrm{~g}$ e o da formulação tradicional foi de $282 \mathrm{kcal} / 100 \mathrm{~g}$. Essa variabilidade no valor calórico ocorreu principalmente em função da diferença no teor de umidade entre as amostras light.

A determinação física da fluidez apresentou variação de 1,0 a $7,5 \mathrm{~cm}$ de escoamento no tempo de 3 minutos (Figura 2). As geleias que se mostraram mais fluidas foram as light, destacando-se as formulações que apresentam esteviosídeo em sua composição (Esteviosídeo F11, Esteviosídeo F8 e F5). Dentre as geleias que apresentaram menor fluidez encontram-se as formulações light contendo sacarina (F2) e ciclamato (F3) isoladamente e em associação (F6). 


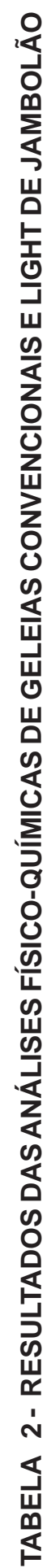

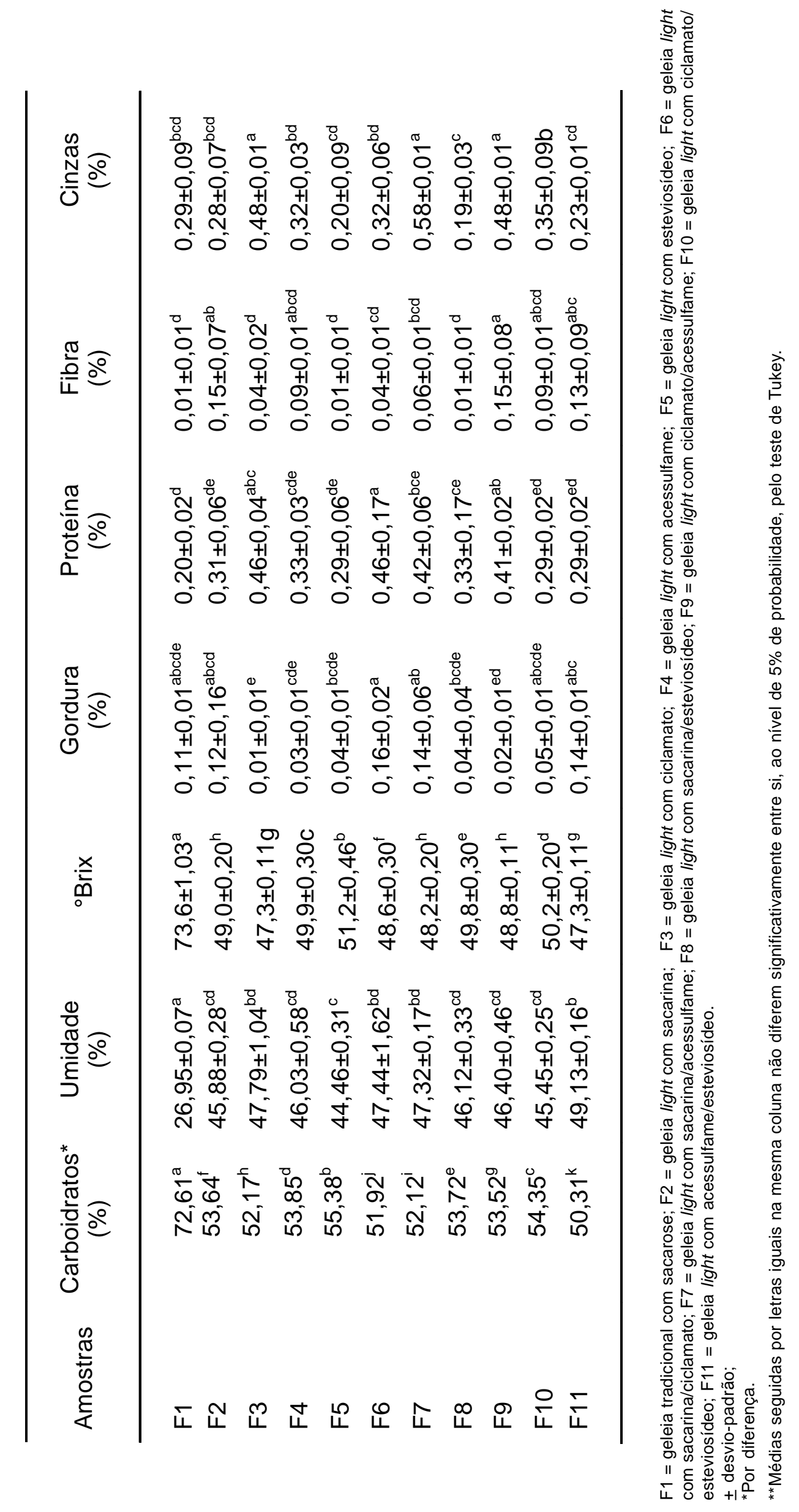




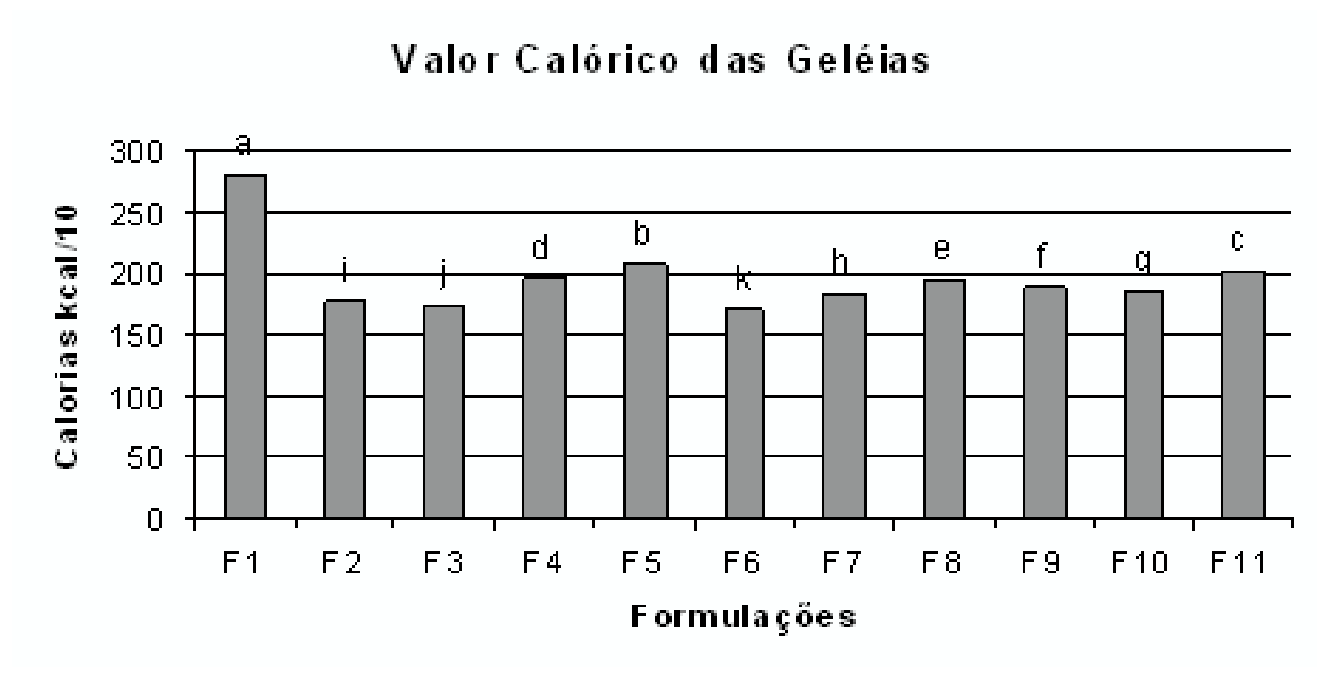

FIGURA 1 - VALOR CALÓRICO DAS GELEIAS DE JAMBOLÃO

F1 = geleia tradicional com sacarose; F2 = geleia light com Sacarina; F3 = geleia light com Ciclamato; F4 = geleia light com acessulfame; F5 = geleia light com esteviosídeo; F6 = geleia light com sacarina/ciclamato; F7 = geleia light com sacarina/ acessulfame; F8 = geleia light com sacarina/esteviosídeo; F9 = geleia light com ciclamato/acessulfame; F10 = geleia light com ciclamato/esteviosídeo; F11 = geleia light com acessulfame/esteviosídeo. Letras iguais entre as colunas não diferem significativamente entre si, ao nível de $5 \%$ de probabilidade, pelo teste de Tukey.

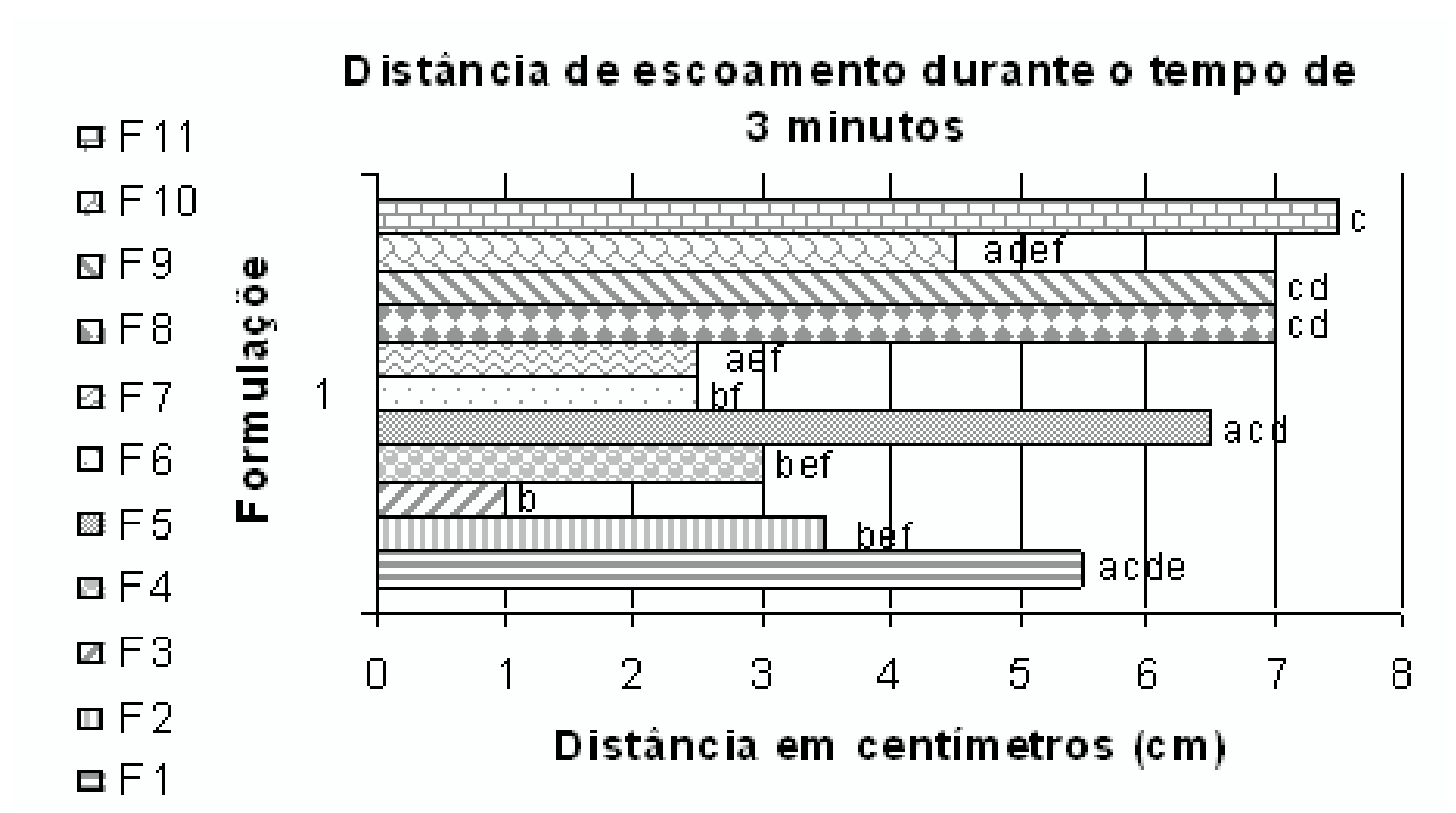

\section{FIGURA 2 - DISTÂNCIA DE ESCOAMENTO (cm) DURANTE O TEMPO DE 3 MINUTOS DAS GELEIAS DE JAMBOLÃO}

F1 = geleia tradicional com sacarose; F2 = geleia light com sacarina; F3 = geleia light com ciclamato; F4 = geleia light com acessulfame; F5 = geleia light com esteviosídeo; F6 = geleia light com sacarina/ciclamato; F7 = geleia light com sacarina/ acessulfame; F8 = geleia light com sacarina/esteviosídeo; F9 = geleia light com ciclamato/acessulfame; F10 = geleia light com ciclamato/esteviosídeo; F11 = geleia light com acessulfame/esteviosídeo. Letras iguais entre as colunas não diferem significativamente entre si, ao nível de $5 \%$ de probabilidade, pelo teste de Tukey. 


\subsection{DETERMINAÇÕES FÍSICO-QUÍMICAS DAS GELEIAS DURANTE O PERÍODO DEARMAZENAMENTO}

Na Tabela 3 estão dispostos os resultados obtidos nas análises físico-químicas, nos tempos 0,30 e 60 dias de armazenamento, das geleias tradicional e light de jambolão.

\section{TABELA 3 - DETERMINAÇÕES FÍSICO-QUÍMICAS DAS GELEIAS DE JAMBOLÃO DURANTE O PERÍODO DE ARMAZENAMENTO}

\begin{tabular}{|c|c|c|c|c|c|c|}
\hline Amostras & Tempo & $\begin{array}{l}\text { Açúcares totais } \\
\text { (\% em glicose) }\end{array}$ & $\begin{array}{c}\text { Açúcares } \\
\text { redutores } \\
\text { (\% em glicose) }\end{array}$ & $\begin{array}{l}\text { Sólidos solúveis } \\
\left.\text { ( }{ }^{\circ} \text { Brix }\right)\end{array}$ & $\mathrm{pH}$ & $\begin{array}{c}\text { Acidez } \\
(\%)\end{array}$ \\
\hline & 0 & $\underline{70,14 \pm 0,65^{a}}$ & $\underline{17,80 \pm 0,21^{\mathrm{C}}}$ & $\underline{73,0 \pm 0,01^{b}}$ & ${\underline{3,71 \pm 0,01^{a}}}^{\mathrm{a}}$ & $\underline{11,75 \pm 0,87^{\mathrm{a}}}$ \\
\hline \multirow[t]{3}{*}{ F1 } & 30 & $68,52 \pm 0,01^{b}$ & $20,29 \pm 0,16^{a}$ & $73,0 \pm 0,01^{b}$ & $3,66 \pm 0,01^{b}$ & $11,73 \pm 0,06^{\mathrm{a}}$ \\
\hline & 60 & $68,14 \pm 0,11^{b}$ & $21,13 \pm 0,37^{b}$ & $74,8 \pm 0,01^{a}$ & $3,67 \pm 0,01^{b}$ & $11,03 \pm 0,06^{\circ}$ \\
\hline & 0 & $\underline{44,63 \pm 0,01^{a}}$ & $\underline{9,99 \pm 0,01^{c}}$ & $\underline{49,0 \pm 0,01^{c}}$ & $\underline{3,74 \pm 0,01^{b}}$ & $9,88 \pm 0,12^{\mathrm{a}}$ \\
\hline \multirow[t]{3}{*}{$\mathrm{F} 2$} & 30 & $42,99 \pm 0,07^{c}$ & $14,25 \pm 0,07^{b}$ & $49,2 \pm 0,01^{b}$ & $3,82 \pm 0,07^{a}$ & $9,12 \pm 0,07^{c}$ \\
\hline & 60 & $44,43 \pm 0,07^{b}$ & $15,31 \pm 0,07^{\mathrm{a}}$ & $48,8 \pm 0,01^{a}$ & $3,72 \pm 0,07^{b}$ & $9,20 \pm 0,07^{b}$ \\
\hline & 0 & $\underline{43,47 \pm 1,89^{a}}$ & ${\underline{8,61 \pm 0,05^{c}}}^{c}$ & $\underline{47,2 \pm 0,01^{a}}$ & $\underline{3,67 \pm 0,01^{a}}$ & $\underline{8,80 \pm 0,04}^{b}$ \\
\hline \multirow[t]{3}{*}{ F3 } & 30 & $39,53 \pm 0,51^{a}$ & $11,86 \pm 0,22^{b}$ & $47,4 \pm 0,01^{b}$ & $3,61 \pm 0,01^{b}$ & $9,66 \pm 0,24^{a}$ \\
\hline & 60 & $40,37 \pm 0,48^{\mathrm{a}}$ & $15,01 \pm 0,38^{\mathrm{a}}$ & $47,4 \pm 0,01^{b}$ & $3,55 \pm 0,01^{c}$ & $8,80 \pm 0,03^{b}$ \\
\hline & 0 & $\underline{49,24 \pm 0,28^{a}}$ & $\underline{9,08 \pm 0,01^{c}}$ & $\underline{50,2 \pm 0,01^{a}}$ & $\underline{3,75 \pm 0,01^{a}}$ & ${\underline{9,38 \pm 0,17^{a}}}^{a}$ \\
\hline \multirow[t]{3}{*}{ F4 } & 30 & $47,53 \pm 0,95^{a b}$ & $12,96 \pm 0,71^{b}$ & $49,6 \pm 0,01^{\mathrm{c}}$ & $3,77 \pm 0,01^{a}$ & $9,49 \pm 0,13^{\mathrm{a}}$ \\
\hline & 60 & $44,33 \pm 1,05^{b}$ & $15,51 \pm 0,48^{\mathrm{a}}$ & $49,9 \pm 0,01^{b}$ & $3,75 \pm 0,01^{a}$ & $9,88 \pm 0,01^{a}$ \\
\hline & 0 & $\underline{51,93 \pm 0,68^{a}}$ & $\underline{10,04 \pm 0,20^{c}}$ & $\underline{51,8 \pm 0,01^{a}}$ & $\underline{3,83 \pm 0,01^{a}}$ & $\underline{9,11 \pm 0,01^{c}}$ \\
\hline \multirow[t]{3}{*}{ F5 } & 30 & $48,18 \pm 1,07^{b}$ & $13,31 \pm 0,62^{b}$ & $51,0 \pm 0,01^{b}$ & $3,74 \pm 0,01^{b}$ & $9,47 \pm 0,10^{b}$ \\
\hline & 60 & $47,48 \pm 0,67^{b}$ & $17,58 \pm 0,69^{a}$ & $51,0 \pm 0,01^{b}$ & $3,63 \pm 0,01^{c}$ & $9,79 \pm 0,04^{\mathrm{a}}$ \\
\hline & 0 & $\underline{42,84 \pm 0,85^{b}}$ & $8,21 \pm 0,25^{c}$ & $49,0 \pm 0,01^{a}$ & $\underline{3,44 \pm 0,01^{c}}$ & ${\underline{9,84 \pm 0,09^{a}}}^{a}$ \\
\hline \multirow[t]{3}{*}{ F6 } & 30 & $38,69 \pm 0,31^{c}$ & $11,63 \pm 0,06^{b}$ & $48,4 \pm 0,01^{c}$ & $3,83 \pm 0,01^{a}$ & $8,70 \pm 0,33^{b}$ \\
\hline & 60 & $48,53 \pm 0,41^{a}$ & $17,78 \pm 0,08^{\mathrm{a}}$ & $48,6 \pm 0,01^{b}$ & $3,78 \pm 0,01^{b}$ & $8,79 \pm 0,01^{b}$ \\
\hline & 0 & $\underline{45,72 \pm 0,97^{a}}$ & $\underline{10,25 \pm 0,25^{c}}$ & $48,2 \pm 0,01^{b}$ & ${\underline{3,64 \pm 0,01^{a}}}^{a}$ & $8,17 \pm 0,02^{a}$ \\
\hline \multirow[t]{3}{*}{ F7 } & 30 & $46,59 \pm 0,82^{a}$ & $13,20 \pm 0,59^{b}$ & $48,0 \pm 0,01^{b}$ & $\overline{3,83 \pm 0,01^{b}}$ & $8,90 \pm 0,28^{b}$ \\
\hline & 60 & $47,04 \pm 0,01^{a}$ & $16,70 \pm 0,51^{a}$ & $48,4 \pm 0,01^{a}$ & $3,81 \pm 0,01^{b}$ & $8,88 \pm 0,01^{b}$ \\
\hline & 0 & ${\underline{48,98 \pm 0,52^{a}}}^{\mathrm{a}}$ & ${\underline{9,44 \pm 0,09^{a}}}^{\mathrm{a}}$ & $49,8 \pm 0,01^{b}$ & $\underline{3,81 \pm 0,01^{b}}$ & ${\underline{8,66 \pm 0,39^{a}}}^{a}$ \\
\hline \multirow[t]{3}{*}{ F8 } & 30 & $44,49 \pm 2,29^{a}$ & $10,45 \pm 0,07^{\mathrm{a}}$ & $49,6 \pm 0,01^{c}$ & $3,80 \pm 0,01^{b}$ & $9,52 \pm 0,50^{a}$ \\
\hline & 60 & $42,70 \pm 1,86^{a}$ & $13,50 \pm 1,81^{a}$ & $50,2 \pm 0,01^{a}$ & $3,69 \pm 0,01^{\mathrm{a}}$ & $8,90 \pm 0,02^{a}$ \\
\hline & 0 & $47,40 \pm 0,42^{\mathrm{a}}$ & $10,36 \pm 0,26^{a}$ & $49,0 \pm 0,01^{a}$ & $\underline{3,78 \pm 0,01^{b}}$ & $9,32 \pm 0,34^{\mathrm{a}}$ \\
\hline \multirow[t]{3}{*}{ F9 } & 30 & $47,00 \pm 0,77^{\mathrm{a}}$ & $14,71 \pm 0,62^{b}$ & $48,8 \pm 0,01^{b}$ & $3,77 \pm 0,01^{b}$ & $9,90 \pm 0,75^{a}$ \\
\hline & 60 & $45,38 \pm 0,49^{\mathrm{a}}$ & $15,56 \pm 0,63^{b}$ & $48,8 \pm 0,01^{b}$ & $3,63 \pm 0,01^{\mathrm{a}}$ & $9,87 \pm 0,04^{a}$ \\
\hline & 0 & $\underline{46,28 \pm 0,27^{a}}$ & $11,45 \pm 0,08^{\mathrm{C}}$ & $50,0 \pm 0,01^{c}$ & $\underline{3,81 \pm 0,01^{a}}$ & ${\underline{9,69 \pm 0,45^{a}}}^{a}$ \\
\hline \multirow[t]{3}{*}{ F10 } & 30 & $51,30 \pm 2,51^{a}$ & $15,54 \pm 0,30^{b}$ & $50,4 \pm 0,01^{a}$ & $3,76 \pm 0,01^{b}$ & $9,61 \pm 0,43^{a}$ \\
\hline & 60 & $45,24 \pm 0,68^{a}$ & $17,92 \pm 0,03^{\mathrm{a}}$ & $50,2 \pm 0,01^{b}$ & $3,72 \pm 0,01^{c}$ & $9,92 \pm 0,04^{\mathrm{a}}$ \\
\hline & 0 & $\underline{50,47 \pm 0,01^{a}}$ & ${\underline{9,90 \pm 0,13^{a}}}^{\mathrm{a}}$ & $47,2 \pm 0,01^{a}$ & $\underline{3,85 \pm 0,01^{a}}$ & $8,68 \pm 0,32^{a}$ \\
\hline \multirow[t]{2}{*}{ F11 } & 30 & $45,36 \pm 1,03^{b}$ & $14,13 \pm 0,43^{b}$ & $47,4 \pm 0,01^{b}$ & $3,69 \pm 0,01^{c}$ & $8,99 \pm 0,06^{a}$ \\
\hline & 60 & $42,85 \pm 0,04^{c}$ & $15,17 \pm 0,03^{b}$ & $47,4 \pm 0,01^{b}$ & $3,72 \pm 0,01^{b}$ & $8,89 \pm 0,03^{a}$ \\
\hline
\end{tabular}

$\mathrm{F} 1$ = geleia tradicional com sacarose; F2 = geleia light com sacarina; F3 = geleia light com ciclamato; F4 = geleia light com acessulfame; F5 = geleia light com esteviosídeo; F6 = geleia light com sacarina/ciclamato; F7 = geleia light com sacarina/acessulfame; F8 = geleia light com sacarina/esteviosídeo; F9 = geleia light com ciclamato/acessulfame; F10 = geleia light com ciclamato/esteviosídeo; F11 = geleia light com acessulfame/esteviosídeo. + desvio-padrão. Médias seguidas por letras iguais na mesma coluna, para cada geléia, não diferem significativamente entre si, ao nível de 5\% de probabilidade, pelo teste de Tukey. 
Praticamente não se verificou variabilidade no conteúdo de sólidos solúveis, acidez e no valor de $\mathrm{pH}$ durante o tempo de armazenamento das geleias, indicando sua estabilidade em relação ao grau de dissociação de ácidos orgânicos. Mota (2007), também não observou variações de pH durante o armazenamento das geleias light de amora-preta, porém os valores de $\mathrm{pH}$ obtidos por esse autor são inferiores (em torno de 3,5) aos encontrados no presente artigo (em torno de 3,7).

A formulação tradicional revelou a menor proporção em açúcares redutores, ficando em cerca de $12,5 \%$ do total de açúcares. As formulações light apresentaram proporções que variaram de $18,5 \%$ a $24,5 \%$. Assim, mesmo com menor tempo de processamento ocorreu maior hidrólise dos açúcares durante o processo de elaboração das geleias light. O conteúdo de açúcares redutores aumentou durante o período de estocagem para todas as formulações. No entanto, observou-se menor taxa de degradação dos açúcares nas formulações light (de 13 a 17\% entre todas as formulações aos 60 dias de armazenamento) em relação a formulação tradicional (19\% de degradação aos 60 dias de armazenamento). Embora a formulação tradicional contenha menor teor de umidade e pH similar ao das formulações light, seu maior teor de acidez pode elevar a taxa de hidrólise dos açúcares.

Durante a hidrólise dos açúcares podem ter ocorrido reações com a formação de compostos de cor escura, intensificando a coloração das geleias. Essas alterações foram percebidas sensorialmente pelos julgadores nas formulações com sacarina (F2), com ciclamato (F3), com sacarina e acessulfame (F7), com sacarina e esteviosídeo (F8) e com acessulfame e esteviosídeo (F11) para as quais foi atribuída intensificação na coloração. Porém, não foi possível associar a taxa maior ou menor de degradação de açúcares em função dos edulcorantes utilizados.

\subsection{AVALIAÇÃO SENSORIAL DAS GELEIAS DE JAMBOLÃO}

Os dados da avaliação sensorial das geleias tradicional e light de jambolão estão expostos na Tabela 4. A geleia tradicional (F1) foi a que apresentou a coloração mais intensa (roxo regular a roxo escuro) quando comparada com as geleias light, provavelmente devido ao maior conteúdo de sacarose e pelo maior tempo de aquecimento durante seu processamento. Dentre as geleias light não se observou influência acentuada sobre a coloração em função do tipo de edulcorante utilizado. As notas conferidas ao atributo cor passaram por alterações durante o período de estocagem para a maioria das geleias, ocorrendo pequeno acréscimo da percepção sensorial na intensidade da cor durante o período de estocagem em quase metade das formulações. $O$ aumento da percepção da cor em algumas geleias pode estar, pelo menos parcialmente, relacionado com pequenas alterações da acidez durante 0 armazenamento. O leve acréscimo da acidez nas formulações contendo ciclamato (F3), sacarina/ acessulfame (F7), sacarina/esteviosídeo (F8) e acessulfame/esteviosídeo (F11) pode ter influenciado a variação da coloração original das antocianinas presentes no jambolão, que em meio ácido assume coloração azulada mais pronunciada que a vermelha.

Os edulcorantes, nas proporções utilizadas neste estudo, inferiram na percepção de doçura das geleias Light, ocorrendo algumas modificações nesse atributo durante o tempo de armazenamento, como o aumento na percepção de doçura pelos julgadores em algumas formulações (F2, F4, F8, F10 e F11) durante esse período. Alguns autores afirmam que a mistura de dois edulcorantes pode beneficiar o perfil de doçura, proporcionando maior facilidade de detecção e maior duração da doçura (BORNSTEIN, WIET e POMBO, 1993). No entanto, neste estudo não se evidenciou alteração da doçura em função do tipo ou da associação de edulcorantes utilizados.

As geleias light foram sensorialmente classificadas como "consistentes", portanto, apresentaram consistência adequada ao tipo de produto. No entanto, na maioria das geleias light ocorreu redução na percepção da consistência pelos julgadores durante o período de armazenamento, indicando pequena perda de rigidez do gel. De acordo com as notas atribuídas pelos julgadores, a geleia tradicional apresentou consistência um pouco mais "firme" que as demais durante os 60 dias de armazenamento. Porém, quando comparada com a análise física de fluidez, a geleia tradicional não demonstrou menor escoamento que a maioria das geleias light (F2, F3, F4, F6, F7, F10). Embora tenha ocorrido variabilidade 
na fluidez (medida fisicamente) das geleias light, essa diferença não foi percebida pelos julgadores na análise sensorial de consistência.

O atributo "sabor residual" atingiu valores na faixa entre 3,0 a 7,2 da escala (de imperceptível a muito intenso), ocorrendo variações durante o tempo de armazenamento em todas as geleias analisadas. Dentre as geleias light em que se utilizou apenas um edulcorante, somente a formulação com ciclamato (F3) não apresentou sabor residual acentuado. Nas geleias com associação dos edulcorantes, as formulações contendo ciclamato (F6, F9 e F10) não revelaram aumento do sabor residual durante $o$ armazenamento. $O$ ciclamato apresenta a vantagem particular de reduzir o gosto amargo residual da sacarina (WELLS, 1989), e provavelmente, também pode ter influenciado positivamente o sabor residual das outras formulações em que estava presente. As geleias contendo esteviosídeo, tanto isolado (F5) como em combinações (F8 e F11), apresentaram aumento da percepção do sabor residual durante o armazenamento. O esteviosídeo tem sido apontado na literatura pelo sabor doce, mas sempre seguido de forte sabor amargo residual (GOTO e CLEMENTE, 1995).

\section{TABELA 4 - DADOS DOS ATRIBUTOS SENSORIAIS DAS GELEIAS DE JAMBOLÃO DURANTE O PERÍODO DE ARMAZENAMENTO}

\begin{tabular}{|c|c|c|c|c|c|c|}
\hline Formulações & $\begin{array}{r}\text { Tempo } \\
\text { (dias) }\end{array}$ & Cor & Doçura & Consistência & Acidez & $\begin{array}{l}\text { Sabor } \\
\text { residual }\end{array}$ \\
\hline F1 & $\begin{array}{c}0 \\
30 \\
60\end{array}$ & $\begin{array}{l}7,3^{\mathrm{a}} \\
7,9^{\mathrm{a}} \\
7,1^{\mathrm{a}}\end{array}$ & $\begin{array}{l}8,0^{\mathrm{a}} \\
7,7^{\mathrm{ab}} \\
7,4^{\mathrm{b}}\end{array}$ & $\begin{array}{l}8,3^{\mathrm{a}} \\
8,0^{\mathrm{a}} \\
7,9^{\mathrm{a}}\end{array}$ & $\begin{array}{l}6,5^{\mathrm{a}} \\
7,1^{\mathrm{a}} \\
7,1^{\mathrm{a}}\end{array}$ & $\begin{array}{l}7,0^{\mathrm{a}} \\
6,8^{\mathrm{a}} \\
5,0^{\mathrm{b}}\end{array}$ \\
\hline $\mathrm{F} 2$ & $\begin{array}{c}0 \\
30 \\
60 \\
\end{array}$ & $\begin{array}{l}5,8^{\mathrm{a}} \\
6,4^{\mathrm{a}} \\
6,4^{\mathrm{a}} \\
\end{array}$ & $\begin{array}{l}5,7^{\mathrm{b}} \\
6,8^{\mathrm{a}} \\
6,3^{\mathrm{ab}}\end{array}$ & $\begin{array}{l}5,8^{\mathrm{a}} \\
6,5^{\mathrm{a}} \\
6,4^{\mathrm{a}} \\
\end{array}$ & $\begin{array}{l}5,2^{b} \\
6,5^{a} \\
5,3^{b}\end{array}$ & $\begin{array}{l}5,2^{b} \\
6,9^{a} \\
6,1^{a} \\
\end{array}$ \\
\hline F3 & $\begin{array}{c}0 \\
30 \\
60\end{array}$ & $\begin{array}{l}4,9^{\mathrm{c}} \\
6,3^{\mathrm{a}} \\
5,6^{\mathrm{b}}\end{array}$ & $\begin{array}{l}7,2^{\mathrm{a}} \\
6,8^{\mathrm{ab}} \\
6,5^{\mathrm{b}}\end{array}$ & $\begin{array}{l}6,9^{a} \\
6,7^{a} \\
5,7^{b} \\
\end{array}$ & $\begin{array}{l}4,7^{\mathrm{c}} \\
6,3^{\mathrm{a}} \\
5,2^{\mathrm{b}} \\
\end{array}$ & $\begin{array}{l}6,6^{a} \\
6,2^{a} \\
5,0^{b} \\
\end{array}$ \\
\hline $\mathrm{F} 4$ & $\begin{array}{c}0 \\
30 \\
60\end{array}$ & $\begin{array}{l}6,6^{a} \\
6,0^{a b} \\
5,3^{b}\end{array}$ & $\begin{array}{l}6,5^{\mathrm{ab}} \\
6,2^{\mathrm{b}} \\
7,1^{\mathrm{a}}\end{array}$ & $\begin{array}{l}7,0^{\mathrm{a}} \\
7,0^{\mathrm{a}} \\
6,4^{\mathrm{b}}\end{array}$ & $\begin{array}{l}5,9^{a} \\
5,3^{b} \\
6,5^{a}\end{array}$ & $\begin{array}{c}4,9^{a b} \\
4,0^{b} \\
5,7^{a}\end{array}$ \\
\hline F5 & $\begin{array}{c}0 \\
30 \\
60\end{array}$ & $\begin{array}{l}6,4^{\mathrm{a}} \\
6,1^{\mathrm{a}} \\
6,0^{\mathrm{a}}\end{array}$ & $\begin{array}{l}7,8^{a} \\
6,5^{b} \\
7,6^{a}\end{array}$ & $\begin{array}{l}7,1^{\mathrm{a}} \\
6,1^{\mathrm{b}} \\
6,0^{\mathrm{b}}\end{array}$ & $\begin{array}{l}5,3^{\mathrm{a}} \\
6,0^{\mathrm{b}} \\
7,0^{\mathrm{c}}\end{array}$ & $\begin{array}{l}5,6^{a} \\
4,6^{b} \\
6,0^{a}\end{array}$ \\
\hline F6 & $\begin{array}{c}0 \\
30 \\
60 \\
\end{array}$ & $\begin{array}{l}6,7^{\mathrm{a}} \\
6,0^{\mathrm{b}} \\
6,3^{\mathrm{b}}\end{array}$ & $\begin{array}{l}7,3^{\mathrm{a}} \\
6,1^{\mathrm{b}} \\
7,0^{\mathrm{a}} \\
\end{array}$ & $\begin{array}{l}5,8^{b} \\
6,8^{a} \\
5,7^{b}\end{array}$ & $\begin{array}{l}6,8^{\mathrm{a}} \\
5,0^{\mathrm{c}} \\
6,0^{\mathrm{b}} \\
\end{array}$ & $\begin{array}{l}6,2^{\mathrm{a}} \\
5,7^{\mathrm{b}} \\
6,5^{\mathrm{a}} \\
\end{array}$ \\
\hline F7 & $\begin{array}{c}0 \\
30 \\
60\end{array}$ & $\begin{array}{l}5,0^{b} \\
5,0^{b} \\
6,2^{a}\end{array}$ & $\begin{array}{l}5,4^{\mathrm{c}} \\
7,0^{\mathrm{a}} \\
6,3^{\mathrm{b}}\end{array}$ & $\begin{array}{l}5,3^{b} \\
4,1^{c} \\
6,0^{a}\end{array}$ & $\begin{array}{l}5,5^{b} \\
5,8^{a} \\
6,1^{a}\end{array}$ & $\begin{array}{l}6,0^{a} \\
4,6^{b} \\
5,4^{a}\end{array}$ \\
\hline F8 & $\begin{array}{c}0 \\
30 \\
60 \\
\end{array}$ & $\begin{array}{l}5,4^{\mathrm{b}} \\
6,0^{\mathrm{a}} \\
6,1^{\mathrm{a}}\end{array}$ & $\begin{array}{l}5,3^{b} \\
6,0^{a} \\
6,1^{a}\end{array}$ & $\begin{array}{l}5,6^{\mathrm{bc}} \\
6,6^{\mathrm{ac}} \\
6,3^{\mathrm{c}}\end{array}$ & $\begin{array}{l}6,2^{\mathrm{a}} \\
5,1^{\mathrm{b}} \\
6,2^{\mathrm{a}}\end{array}$ & $\begin{array}{l}4,3^{b} \\
5,1^{b} \\
6,7^{a}\end{array}$ \\
\hline F9 & $\begin{array}{c}0 \\
30 \\
60\end{array}$ & $\begin{array}{l}6,0^{\mathrm{a}} \\
5,1^{\mathrm{b}} \\
5,4^{\mathrm{b}}\end{array}$ & $\begin{array}{l}7,1^{\mathrm{a}} \\
6,6^{\mathrm{a}} \\
6,3^{\mathrm{a}} \\
\end{array}$ & $\begin{array}{l}6,6^{\mathrm{a}} \\
6,5^{\mathrm{a}} \\
6,0^{\mathrm{a}}\end{array}$ & $\begin{array}{l}6,7^{\mathrm{a}} \\
5,8^{\mathrm{b}} \\
6,3^{\mathrm{a}}\end{array}$ & $\begin{array}{l}6,1^{\mathrm{a}} \\
4,9^{\mathrm{bc}} \\
5,3^{\mathrm{a}}\end{array}$ \\
\hline F10 & $\begin{array}{c}0 \\
30 \\
60\end{array}$ & $\begin{array}{l}5,7^{\mathrm{a}} \\
5,9^{\mathrm{a}} \\
5,8^{\mathrm{a}}\end{array}$ & $\begin{array}{l}6,0^{\mathrm{a}} \\
6,2^{\mathrm{a}} \\
6,7^{\mathrm{a}} \\
\end{array}$ & $\begin{array}{l}7,0^{\mathrm{a}} \\
6,9^{\mathrm{a}} \\
6,1^{\mathrm{b}}\end{array}$ & $\begin{array}{l}6,1^{\mathrm{a}} \\
5,5^{\mathrm{b}} \\
6,3^{\mathrm{a}}\end{array}$ & $\begin{array}{l}7,2^{\mathrm{a}} \\
5,1^{\mathrm{b}} \\
6,3^{\mathrm{a}}\end{array}$ \\
\hline F11 & $\begin{array}{c}0 \\
30 \\
60 \\
\end{array}$ & $\begin{array}{l}4,8^{\mathrm{b}} \\
5,3^{\mathrm{a}} \\
5,4^{\mathrm{a}}\end{array}$ & $\begin{array}{l}5,7^{b} \\
6,5^{c} \\
7,1^{a} \\
\end{array}$ & $\begin{array}{l}5,0^{b} \\
6,2^{a} \\
6,1^{a}\end{array}$ & $\begin{array}{l}5,7^{b} \\
6,4^{a} \\
6,2^{a} \\
\end{array}$ & $\begin{array}{l}5,8^{\mathrm{b}} \\
5,4^{\mathrm{b}} \\
7,0^{\mathrm{a}}\end{array}$ \\
\hline
\end{tabular}

$\mathrm{F} 1$ = geleia tradicional com sacarose; F2 = geleia light com sacarina; F3 = geleia light com ciclamato; F4 = geleia light com acessulfame; F5 = geleia light com esteviosídeo; F6 = geleia light com sacarina/ciclamato; F7 = geleia light com sacarina/acessulfame; F8 = geleia light com sacarina/esteviosídeo; F9 = geleia light com ciclamato/acessulfame; $\mathrm{F} 10$ = geleia light com ciclamato/esteviosídeo; F11 = geleia light com acessulfame/esteviosídeo. Médias seguidas por letras iguais para cada geleia na mesma coluna não diferem entre si pelo teste de Tukey ao nível de $5 \%$ de probabilidade. 
Mesmo em combinações, exceto com ciclamato (F10), o esteviosídeo manteve seu sabor residual característico, afetando negativamente o sabor residual das geleias. Ao sabor residual da geleia tradicional foi atribuída a média 7,0 no tempo zero, provavelmente pelo sabor da fruta ser pouco conhecido em razão do pequeno consumo do jambolão na forma "in natura". O uso dos edulcorantes mascarou parcialmente o sabor residual em relação à formulação tradicional (F1), afetando positivamente o sabor das geleias light de jambolão.

\section{CONCLUSÃO}

Todas as geleias apresentam características físico-químicas de acordo com os padrões da legislação brasileira, as quais se mantiveram estáveis durante o tempo de estocagem, com exceção da hidrólise parcial de açúcares não redutores que se mostrou superior na geleia tradicional.

A geleia tradicional manteve suas características sensoriais estáveis durante o tempo de armazenamento, diferentemente das geleias light que apresentaram acréscimo nos valores de doçura e redução na consistência.

Não se observou influência do tipo de edulcorante utilizado nas formulações e de suas combinações nas características físico-químicas entre as diferentes formulações light. As combinações de edulcorantes também não ocasionaram diferenças sensoriais nos produtos quando comparadas às formulações que continham apenas um tipo de edulcorante.

\section{ABSTRACT}

\section{PHYSICO-CHEMICAL AND SENSORY CHARACTERISTICS OF JAMBOLAN JELLIES}

The aim of this work was to use jambolan fruit to elaborate conventional and light jellies, and evaluate its physico-chemical and sensory stability during 60 days storage. In the light jellies formulation were used four kinds of sweeteners, isolated and combined: saccharin, cyclamate, acessulfame-k and stevioside. The physico-chemical analysis carried out after the process were fluidity, moisture, lipids, protein, fiber, ash, carbohydrates (total and reduced), total soluble solids, $\mathrm{pH}$ and total acidity of the products. The results showed that the physico-chemical characteristics of all the jellies were appropriated according to the Brazilian Law patterns, and the characteristics were stable during the storage period. At the sensory analysis, the light formulations did not become stable only to the sweetness and consistency characteristics. The use of sweeteners and its combinations in the formulation of light jellies did not result on any sensory interference.

KEY-WORDS: JAMBOLAN JELLIES; JAMBOLAN; LIGHT JELLIES.

\section{REFERÊNCIAS}

1 ABNT. Associação Brasileira de Normas Técnicas. NBR 12994: métodos de avaliação sensorial de alimentos e bebidas. Rio de Janeiro, 1993. 2 p.

2 ÁRVORES medicinais.On line. Disponível em:<http://www.home/arvores_medicinais/ arvores_medicinais.htm>. Acesso em: 15 de jul. 2005.

3 BORNSTEIN, B. L.; WIET, S. G.; POMBO, M. Sweetness adaptation of some carbohydrate and high potency sweeteners. Journal of Food Science, Chicago, v. 58, n.3, p.595-598, 1993.

4 BRASIL. Ministério da Saúde. Agência de Vigilância Sanitária. Resolução RDC nº 18, de 24 de março de 2008. Regulamento técnico que autoriza o uso de aditivos edulcorantes em alimentos, com seus respectivos limites máximos. Diário Oficial [da] República Federativa do Brasil, Brasília 25 de março de 2008.

5 BRASIL. Ministério da Saúde. Agência de Vigilância Sanitária. Resolução RDC nº 360, de 23 de dezembro de 2003. Regulamento técnico sobre rotulagem nutricional de alimentos embalados, tornando obrigatória a rotulagem nutricional. Diário Oficial [da] República Federativa do Brasil, Brasília, n.57-E, 2001.

6 BRASIL. Ministério da Saúde. Portaria $N^{\circ}$ 204, de 4 de maio de 1978. Define termos sobre geléia de frutas. Diário Oficial [da] República Federativa do Brasil, Brasília, n.15-E, 1978. 
7 CAMPOS, A. M.; CÂNDIDO, L. M. B. Formulação e avaliação físico-química e reologia de geléias de baixo teor de sólidos solúveis com diferentes adoçantes e edulcorantes. Ciência e Tecnologia de Alimentos, Campinas, v. 15, n.3, p. 268-278, 1995.

8 CORRÊA, P. Dicionário das plantas úteis do Brasil e das exóticas cultivadas. Rio de Janeiro: Imprensa Nacional/Ministério da Agricultura, 1984. v. 3.

9 GOTO, A.; CLEMENTE, E. Influência do rebaudiosídeo A na solubilidade e no sabor do esteviosídeo. Ciência e Tecnologia de Alimentos, Campinas, v. 18, n.1, p.3-6, 1995.

10 GULARTE, M. A. Manual de análise sensorial de alimentos. Pelotas: Universidade Federal de Pelotas, 2002. 59 p.

11 HIGGINBOTHAM, J. D. Recent developments in non-nutritive sweeteners. In: GREMBY,T. H.; PARKER,K.J.; LINDLEY, M.G. Developments in sweeteners-2. London: Applied Science, 1983. p. 55-119.

12 INSTITUTO ADOLFO LUTZ. Normas analíticas do Instituto Adolfo Lutz: métodos químicos e físicos para análise de alimentos. 3 ed. São Paulo, 1985. v.1.

13 MOTA, R. V. Características químicas e aceitabilidade de geléias de amora-preta de baixo teor de sólidos solúveis. Brazilian Journal of Food Technology, v. 10, n. 2, p. 116-121, 2007.

14 MORTON, J. Fruits of warm climates. Miami: Creative Resource Systems, 1987. p. 375-378.

15 PARKE, S. A.; BIRCH, G. G.; PORTMANN, M. O.; KILCAST, D. A study of the solution properties of selected binary mixture of bulk and intense sweeteners in relation to their psychophysical characteristics. Food Chemistry, Chicago, v. 67, n. 3, p.247-259, 1999.

16 SALGADO, J. M. Adoçantes dietéticos: doçura que não vem da cana. Diário Popular, Pelotas, Caderno Saúde, p.2-3, 04 de novembro de 2001.

17 STATSOFT, INC. Statistica. Versão 7.0. Tulsa, 2008.

18 WELLS, A. G. The use of intense sweeteners in soft drinks. In: GREMBY, T.H. Progress in sweeteners. New York: Elsevier Applied Science, 1989. , p.121-42. 